Portland State University

PDXScholar

$11-22-2019$

\title{
Caring about Aftercare: Thesis Presentation of Initial Findings
}

Sage B. Fuentes

Portland State University

Follow this and additional works at: https://pdxscholar.library.pdx.edu/honorstheses

Part of the Psychology Commons

Let us know how access to this document benefits you.

\section{Recommended Citation}

Fuentes, Sage B., "Caring about Aftercare: Thesis Presentation of Initial Findings" (2019). University Honors Theses. Paper 818.

https://doi.org/10.15760/honors.837

This Thesis is brought to you for free and open access. It has been accepted for inclusion in University Honors Theses by an authorized administrator of PDXScholar. Please contact us if we can make this document more accessible: pdxscholar@pdx.edu. 
Caring about Aftercare:

Thesis Presentation of Initial Findings

Sage Fuentes

Presented to the faculty of

Portland State University 
Abstract

While previous research has studied the impact of stigma and non-normative play within the BDSM and kink community, previous psychological research involving BDSM practitioners has not investigated the cultural practice of aftercare that tends to follow the highly sensationalized acts within the culture. The current study this cultural practice by giving a series of semi-structured interviews to assess the importance of aftercare and how it impacts negotiations and play. BDSM practitioners $(\mathrm{n}=11)$ participated in audio recorded interviews regarding questions on identity, kink, aftercare, consent, and negotiation. $72.73 \%$ of individuals rated aftercare as mostly to largely important in their lives. Aftercare presented itself in a variety of ways including physical affection, verbal affirmation, and acts of service. 
Caring About Aftercare: Thesis Presentation of Initial Findings

Examinations of the diverse cultural formations that humans have created over time have provided great insight into the inner working of humanity as a whole. One such subculture of pop culture and psychological interest has firmly rooted itself around the associated practices and lifestyles of BDSM and kink. BDSM is an initialization standing for bondage/discipline, domination/submission (commonly stylized as D/s), and sadism/masochism (commonly written as S\&M) (Hébert \& Weaver, 2014; Wismeijer \& Marcel, 2013). Kink is a broader term used to describe non-normative sex practices some of which more obviously belong to BDSM (such as use of implements or bondage during sex practice), something that may involve multiple aspects of BDSM (such as role-play), or other fringe sexual or sensual acts (Shahbaz, \& Chirinos, 2017). Kink is also used in reference as a whole to the subculture such as the terms kink event, kink friendly, kinkster (a gender neutral term in reference to a practitioner of kink or BDSM), and so on. In recent times, the practice and subculture around BDSM and kink lifestyles have been recognized closer to the mainstream not just in pop culture, but with an increase in academic analysis about the psychological aspects of subculture practitioners. Stigmatization of kink has deep historical roots and has been studied though a few modes of research and community lead publications (Stiles \& Clark, 2011; Moser \& Madeson, 2002)

BDSM and kink cultures have several practices that lead up to and surround the nonnormative acts for which they have been identified, including aftercare (Shahbaz, \& Chirinos, 2017; Thomas, 2017). Aftercare is the practice of following up a "scene" (any BDSM or kink related play that may or may not involve sex) with physical touch, affirmation, and other forms of care to comfort the taxing acts that can be found in this non-normative play (Shahbaz \& Chirinos, 2017; Thomas, 2017). Currently, no research focusing on aftercare within the kink and BDSM 
community has been conducted in the psychological community.

For this research, individuals in the kink community that have shown interest in participating in research related to aftercare are being interviewed. As there is no current literature that examines aftercare, we choose to examine the construct exploratively. The interview provides to us a narrative to better understand this construct as previous work does not thoroughly exist within the psychological field. The primary benefits of this project are furthering an understanding of the BDSM and kink cultural practice of aftercare and providing a better understanding of the behavior of a subculture that is still only minimally known.

\section{Methods}

\section{Participants}

Participants have been recruited from Portland, OR metro area-based kink-friendly spaces during events. Advertisements of the study have focused on the cultural practices of aftercare. Additionally, advertisements have expressed that participants can earn anywhere from $\$ 15$ to $\$ 45$ for their participation with the ability to donate some proceeds to their associated kink-friendly space from which they were recruited. Participants were asked to arrange a sixty to ninety-minute block of time with the investigator to conduct the interview, inventories, and experiments. A follow up e-mail or meeting was also part of arrangements as to award additional earnings or provide a receipt of donations (as per the procedure). Participants were only considered if the individual had a minimum of two cumulative years in the subculture and are been a current resident of the Portland, OR metro area as to establish experienced individuals in relation to kink and BDSM. All participants are age 18 or older in keeping with legal and ethical practices.

\section{Apparatus/Materials}


Paper print outs have been used for the inventory and choice experiments. Interviews were documented by hand with two audio recording devices used to provide mitigate error.

\section{Procedure}

Each meeting took place in a public space agreed upon by the attending interviewer and the participant. A public space is being considered for the comfortability of subculture individuals given a possibly negative rapport with psychologists due to a previous history of research pointing to BDSM as psychopathological (Shahbaz, \& Chirinos, 2017). The initial meeting was held over a 60 to 90 minute block of time with no deviation in participation fee based on length of time. The base participation fee was $\$ 15$ in keeping with the local minimum wage, potential costs of travel, and the depth of information being asked. Only individuals that participate in the initial meeting qualified for the participation fee or the opportunity to take part in the associated choice experiment and game that are not covered in this paper, but the collected data will exist in a future publication. Consent forms that have been generated detailing the basic premise of the experiment, assessment of risks, and contact information was given out and reviewed with each participant at the beginning of each meeting. Each session was held in the order of interview, prosocial inventory, and choice experiments to maintain consistency across all assessments.

Interview questions were adapted from other material on prosocial behavior to include rapport developing questions based around the lifestyle (See Appendix). Questions directly related to the cultural constructs of aftercare were asked. A 10-point Likert scale for accessing importance of aftercare to personal life was used to standardize responses, one was marked as minimally important, 5 was of average importance, and 10 was marked as being of utmost importance. The interviews were audio recorded for coding accuracy. 


\section{Results/Analysis}

Selected descriptive characteristics of the study participants are presented in Table 1.

\section{Table 1}

Selected Descriptive Characteristics of Participants $(n=11)$

\begin{tabular}{llr}
\hline Categories & $\%$ & N \\
\hline Orientation & & \\
Bi/Pan & 45.45 & 5 \\
Heterosexual/Heteroflexible & 27.27 & 3 \\
Homosexual/Homoflexible & 18.18 & 2 \\
Ace & 9.09 & 1
\end{tabular}

Biological Sex or Assigned Gender at Birth (AGAB)

AFAB/Female

AMAB/Male

Decided not to Declare

Gender Identity

Non-binary

Female

Male

$45.45 \quad 5$

$36.36 \quad 4$

$18.18 \quad 2$

Relationship Type

Polyamorous/Ethically

Relationship/Ambiarmous

Monogamous
Non-monogamous/Open

63.647

$36.36 \quad 4$ 
Due to the intersection of identities within individuals in the kink, many of the descriptive statistics can only give a portion of the reality of the individual's identities. The 11 participants were identified using four open ended questions on orientation, gender or gender identity, biological sex or assigned gender at birth, and relationship type. Orientation had four dominant categories appear: Bi/Pan (five individuals), Heterosexual/Heteroflexible (three individuals), Homosexual/Homoflexible (three individuals), and Ace (1 individual). Gender/Gender Identity had three dominant categories (for individuals that identified with multiple gender identities such as trans* in addition to non-binary, they were placed in the non-binary category): Non-binary (five individuals), Female (four individuals), and Male (two individuals). Biological Sex/Assigned Gender at Birth had two categories with one individual wishing to not declare: AFAB/Female (eight individuals) and AMAB/Male (two individuals). Relationship type was divided into Nonmonogamous (including, but not limited to, polyamorous, Ethically Non-monogamous, Open Relationship, and Ambiamorous) (seven individuals) and Monogamous (four individuals). The term ambiamorous meaning individuals that feel satisfied in either a monogamous or nonmonogamous relationship, largely depending on the individual(s) they are with.

The collection of role identities (roles within kink that an individual aligns themselves with) was a much more difficult metric. Nine out of the 11 individuals provided multiple role identities with the average role per individual being 3.91 roles $(72.73 \%$ of individuals had three roles or more). The following roles were identified in the research sample: Switch (six instances), Pet (six instances), Bottom (three instances), Sub (three instances), Kitten (two instances), Little (two instances), Owner (two instances), Brat (two instances), Dom (two instances), Sensationist (two instances), Mistress (two instances), Babygirl (two instances), Princess (one instance), 
Middle (one instance), Sadist (one instance), Primal (one instance), Mommy (one instance), Verse (one instance), Miss (one instance), Prey (one instance). Some roles were omitted as to not identify individuals that may belong to small subgroups within kink as well.

Responses for how aftercare was performed existed across several questions trying to assess the construct of aftercare. In keeping with the formal definitions that have been found, physical affection and verbal affirmation were commonly reported as methods for aftercare. Other methods of care were broader and included service (such as providing food, water, blankets, or entertainment for one of the individuals in the scene), medical attention when needed (such as in bloodplay or specific acts of electroplay), and either providing conversation or providing isolation. A majority of the sample $(72.73 \%)$ reported that what aftercare looked like between two individuals was partner dependent. A majority of the sample (72.73\%) reported that aftercare rated as mostly or largely important to their personal lives. Additionally, aftercare was reported in all interviews of bottom or submissive identifying individuals as something they give to tops, not just receive from tops.

\section{Discussion}

With this portion of this study, we aimed to increase the depth of understanding on aftercare as a cultural construct. As it stands the robust practice needs a great deal more exploration to understand other benefits especially as we consider statistical tests with sufficient assumption criteria (i.e. appropriate sample sizes). What we can ascertain from the collected data is that aftercare is a diverse practice that matches the similarly diverse demographic that is the individuals in the kink community. The individualized nature of aftercare needs makes for the realization of a much more intimate interaction than may be characterized in the current stigmatization of kink and BDSM, specifically because the acts encompassed in aftercare are acts that are seen much 
more commonly in the vanilla world.

Aftercare appears to exhibit some characteristics traditionally associated with prosocial behavior, namely reciprocity and trust, and as such, it may be a potential marker for prosocial tendencies in keeping with previous research on the diverse forms of prosocial behavior (as cited in Carlo, Hausmann, Christiansen, \& Randall, 2003). The extent that prosocial behavior among BDSM individuals has been analyzed has been limited to self-reported altruism scores using the Self-Report Altruism Scale (SRAS) and have not used broader measures of prosocial behavior such as semi-structured interview or incentivized choice experiment (Hébert \& Weaver, 2014). The current standing is that practitioners of the BDSM lifestyle score lower than normative data comparisons on self-reported inventories of altruism. That analysis looked only at BDSM practitioners interacting with strangers and not with other members of their in-group (Hébert \& Weaver, 2014).

Due to the scope of the research, limitations could not be avoided. Increased sample size would allow for greater randomization to control for possible order effects. Due to difficulty in recruiting participants in an in-person environment, bias was no doubt introduced into the study. The bias likely appears as sampling bias due to advertisement (advertisements for the study related to aftercare, so those with interests in aftercare were more likely to respond), sampling bias due to a private community (people not wishing to divulge information about their personal lives), and sampling bias related to limited physical venues to recruit participants (working with an organization to be allowed to set up recruitment space required rapport and time). Possible interaction effects from settings would be minimized with larger sample sizes due to the belief that disingenuous results would appear in a stricter setting such as a lab.

\section{References}


CARING ABOUT AFTERCARE

Hébert, A., \& Weaver, A. (2014). An examination of personality characteristics associated with BDSM orientations. Canadian Journal of Human Sexuality, 23(2), 106-115. doi:http://dx.doi.org/10.3138/cjhs.2467

Moser, C. and J. Madeson. (2002). Bound to be free: The SM experience. New York: Continuum.

Shahbaz, C., \& Chirinos, P. (2017). Becoming a kink aware therapist. Routledge/Taylor \& Francis Group, New York, NY. Retrieved from http://search.proquest.com/docview/1938692726?accountid=13265

Stiles, B. L., \& Clark, R. E. (2011). BDSM: A subcultural analysis of sacrifices and delights. Deviant Behavior, 32(2), 158-189. doi:http://dx.doi.org/10.1080/01639621003748605

Thomas, S. S. (April 24, 2017). Why aftercare is the BDSM practice that everyone should be doing. Refinery29. Retrieved from https://www.refinery29.com/en-us/what-is-bdsm-sex$\underline{\text { aftercare }}$

Wismeijer, A. A. J., \& van Assen, Marcel A. L. M. (2013). Psychological characteristics of BDSM practitioners. Journal of Sexual Medicine, 10(8), 1943-1952. doi:http://dx.doi.org/10.1111/jsm.12192 
Appendix

Interview questions

1. What do you identify as your sexual orientation?

2. What do you identify as your gender?

3. What do you identify as your biological sex?

4. What would you identify as your romantic and relationship type or types? (some examples include monogamous, polyamorous, ethically non-monogamous, asexual, gray romantic, etc.)

5. What roles, titles, or subgroups of kink do you identify with?

6. What got you interested in kink?

7. Can you tell us about your role or roles in the local scene?

8. Have you volunteered time at all in the past three months? Past year? Past two years?

9. What interested you in participating in an interview on kink and aftercare?

10. Have you ever attended a class on kink?

11. Have you taught a class on kink or mentored anyone in the kink community?

12. Have you donated anything other than time in the last three months? Past year? Past two years?

13. How do you go about vetting a partner for play, either casual play or otherwise?

14. Do you negotiate aftercare before playing with a first time partner?

15. Can you describe your negotiation and consent dialogue process for a first time partner?

16. Do you actively practice aftercare, either giving or receiving?

17. What sort of ways do you give care to others in aftercare?

18. What sort of ways do you prefer for receiving your own aftercare? 
CARING ABOUT AFTERCARE

19. Would you say you have a specific style of giving aftercare?

20. Would you say you participate in any play that requires more than average aftercare, either giving or receiving?

21. On a scale of 1 to 10 , with 1 being minimally important, 5 being of average importance, and 10 being of utmost importance, how important would you say aftercare is to your personal life?

22. Is there anything specifically you would like to say to the research community as a whole as a member of the BDSM or kink community? 